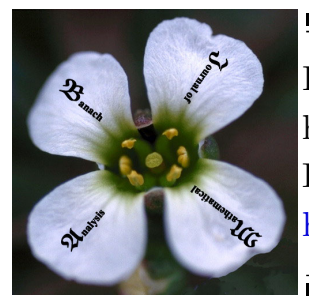

Banach J. Math. Anal. 9 (2015), no. 2, 35-43

http://doi.org/10.15352/bjma/09-2-4

ISSN: $1735-8787$ (electronic)

http://projecteuclid.org/bjma

\title{
WEIGHTED COMPOSITION OPERATORS ON WEAK VECTOR-VALUED BERGMAN SPACES AND HARDY SPACES
}

\author{
MOSTAFA HASSANLOU ${ }^{1 *}$, HAMID VAEZI ${ }^{1}$ AND MAOFA WANG ${ }^{2}$ \\ Communicated by S. Sugawa
}

\begin{abstract}
In this paper we investigate weighted composition operators between weak and strong vector-valued Bergman spaces and Hardy spaces, and give some estimates of their norms.
\end{abstract}

\section{INTRODUCTION}

Let $\mathbb{D}$ be the open unit disk of the complex plane $\mathbb{C}, \varphi$ be an analytic self-map of $\mathbb{D}$; that is $\varphi(\mathbb{D}) \subset \mathbb{D}$, and $u$ a scalar-valued analytic function on $\mathbb{D}$. The weighted composition operator $u C_{\varphi}$ on analytic functions on $\mathbb{D}$ is defined by:

$$
u C_{\varphi}(f)(z)=u(z) f(\varphi(z)) .
$$

When $u(z) \equiv 1$, we just get the composition operator $C_{\varphi}, C_{\varphi}(f)(z)=f \circ \varphi(z)$. Also if $\varphi(z)=z$, the identity function, then we get the multiplication operator $M_{u}, M_{u}(f)(z)=u(z) f(z)$. Various aspects of composition operators have been studied over the past several decades. We refer to monographs by CowenMacCluer [6] and Shapiro [17] for an overview of the work before the mid-1990s.

Recently, as an extension of composition operators, weighted composition operators have been studied quite extensively on different spaces of scalar-valued analytic functions (for example, see $[5,15,16,18,22]$ ). Now there is an interest on studying these operators acting on spaces of vector-valued analytic functions.

Date: Received: Dec. 6, 2013; Revised: May 7, 2014; Accepted: Jun. 3, 2014.

* Corresponding author.

2010 Mathematics Subject Classification. Primary 47B38; Secondary 46E40.

Key words and phrases. Weighted composition operator, vector-valued Bergman space, vector-valued Hardy space, Hilbert-Schmidt operator. 
Weak compactness of composition operators on such analytic vector-valued function spaces were studied in [4, 14]. In [10, 12], Esmaeili and Mahyar, Laitila and Tylli characterized bounded and (weak) compact operator-weighted composition operators on these kind of spaces. Laitila, Tylli and Wang [13] studied composition operators from weak to strong vector-valued Bergman and Hardy spaces. In [21], Wang presented some necessary and sufficient conditions for weighted composition operators to be bounded on vector-valued Dirichlet spaces.

Let $X$ be a complex Banach space and $p \geq 1$. The vector-valued Bergman space $A^{p}(X)$ consists of all analytic functions $f: \mathbb{D} \rightarrow X$ such that

$$
\|f\|_{A^{p}(X)}=\left(\int_{\mathbb{D}}\|f(z)\|_{X}^{p} d A(z)\right)^{\frac{1}{p}}<\infty,
$$

where $d A$ is the normalized area measure on $\mathbb{D}$. Also, the vector-valued Hardy space $H^{p}(X)$ is the set of all analytic functions $f: \mathbb{D} \rightarrow X$ for which

$$
\|f\|_{H^{p}(X)}=\sup _{0<r<1}\left(\int_{\mathbb{T}}\|f(r \zeta)\|_{X}^{p} d m(\zeta)\right)^{\frac{1}{p}}<\infty,
$$

where $d m(\zeta)$ is the normalized Lebesgue measure on the unit circle $\mathbb{T}=\partial \mathbb{D}$. In the case $X=\mathbb{C}$, we write $A^{p}(X)=A^{p}$ and $H^{p}(X)=H^{p}$. By taking $p=2$, the scalar-valued spaces $A^{2}$ and $H^{2}$ become Hilbert spaces. The following weak version of vector-valued spaces were considered by e.g. Blasco [2] and Bonet, Domanski and Lindstrom [4]: the weak spaces $w A^{p}(X)$ and $w H^{p}(X)$ consist of all analytic functions $f: \mathbb{D} \rightarrow X$ for which

$$
\|f\|_{w A^{p}(X)}=\sup _{\left\|x^{*}\right\| \leq 1}\left\|x^{*} \circ f\right\|_{A^{p}}, \quad\|f\|_{w H^{p}(X)}=\sup _{\left\|x^{*}\right\| \leq 1}\left\|x^{*} \circ f\right\|_{H^{p}},
$$

are finite, respectively. Here $x^{*} \in X^{*}$, the dual space of $X$. Refer to $[1,3,13]$ for more information about vector-valued Bergman and Hardy spaces.

It is well known that for every analytic map $\varphi: \mathbb{D} \rightarrow \mathbb{D}$, the operators $C_{\varphi}: A^{p}(X) \rightarrow A^{p}(X)$ and $C_{\varphi}: H^{p}(X) \rightarrow H^{p}(X)$ are bounded, and also $C_{\varphi}$ is bounded on $w A^{p}(X)$ and $w H^{p}(X)$, (for example, see [4] or [14]). We consider the infinite dimensional complex Banach space $X$, since $w A^{p}(X)=A^{p}(X)$ and $w H^{p}(X)=H^{p}(X)$, for $p \geq 1$ and any finite dimensional Banach space $X$. For any infinite dimensional complex Banach space $X, A^{p}(X) \neq w A^{p}(X)\left(H^{p}(X) \neq\right.$ $\left.w H^{p}(X)\right)$ and $\|.\|_{w A^{p}(X)}$ is not equivalent to $\|\cdot\|_{A^{p}(X)}$ on $A^{p}(X)\left(\|\cdot\|_{w H^{p}(X)}\right.$ is not equivalent to $\|.\|_{H^{p}(X)}$ on $\left.H^{p}(X)\right)$, for example, see [13, Proposition 3.1] or [11, Example 15].

Our aim in this paper is, by using the main idea in [13], to estimate the norms of weighted composition operators between $w A^{p}(X)$ and $A^{p}(X)$, and also between $w H^{p}(X)$ and $H^{p}(X)$, for $p \geq 2$. This is motivated by the fact that between scalar function spaces weighted composition operators are usually much more complex than unweighted composition operators. Our results are now generalizations to scalarly weighted composition operators of results from Laitila, Tylli and Wang [13], where unweighted composition operators were considered. As an application, we obtain the corresponding estimate of norm for the multiplication operator. We also see that, for the case $p=2$, the boundedness of the weighted composition 
operator between weak and strong vector-valued Bergman spaces (resp. Hardy spaces) is equivalent to membership in Hilbert-Schmidt class of the operator between the corresponding scalar-valued Bergman space (resp. Hardy space).

In the rest of the paper, letter $c$ will stand for positive constants and not necessarily same in each occurrence. Also, the notation $A \approx B$ means that there is a positive constant $c$ such that $A / c \leq B \leq c A$.

\section{Main Results}

We first give the following basic estimates.

Proposition 2.1. Let $X$ be any complex Banach space and $1 \leq p<\infty$. Then

$$
\left\|u C_{\varphi}: w A^{p}(X) \longrightarrow A^{p}(X)\right\| \leq\left(\int_{\mathbb{D}} \frac{|u(z)|^{p}}{\left(1-|\varphi(z)|^{2}\right)^{2}} d A(z)\right)^{1 / p}
$$

and

$$
\left\|u C_{\varphi}: w H^{p}(X) \longrightarrow H^{p}(X)\right\| \leq \sup _{0<r<1}\left(\int_{\mathbb{T}} \frac{|u(r \zeta)|^{p}}{1-|\varphi(r \zeta)|^{2}} d m(\zeta)\right)^{1 / p} .
$$

Proof. By the well-known inequality from [20, 23] we have

$$
|f(z)| \leq \frac{\|f\|_{A^{p}}}{\left(1-|z|^{2}\right)^{\frac{2}{p}}}
$$

for any $f \in A^{p}$ and $z \in \mathbb{D}$. Thus, for $f \in w A^{p}(X)$, we have

$$
\begin{gathered}
\|f(z)\|_{X}^{p}=\sup _{\left\|x^{*}\right\| \leq 1}\left|\left(x^{*} \circ f\right)(z)\right|^{p} \leq \frac{1}{\left(1-|z|^{2}\right)^{2}} \sup _{\left\|x^{*}\right\| \leq 1}\left\|x^{*} \circ f\right\|_{A^{p}}^{p} \\
=\frac{1}{\left(1-|z|^{2}\right)^{2}}\|f\|_{w A^{p}(X)}^{p} .
\end{gathered}
$$

Hence

$$
\begin{aligned}
\left\|u C_{\varphi} f\right\|_{A^{p}(X)}^{p} & =\int_{\mathbb{D}}|u(z)|^{p}\|f(\varphi(z))\|_{X}^{p} d A(z) \\
& \leq\|f\|_{w A^{p}(X)}^{p} \int_{\mathbb{D}} \frac{|u(z)|^{p}}{\left(1-|\varphi(z)|^{2}\right)^{2}} d A(z) .
\end{aligned}
$$

In the case of Hardy spaces, for any analytic map $f: \mathbb{D} \rightarrow \mathbb{C}$ we have

$$
|f(z)|^{p} \leq \frac{\|f\|_{H^{p}}^{p}}{1-|z|^{2}} \quad z \in \mathbb{D}
$$

see $[9,23]$. If $f \in w H^{p}(X)$, then

$$
\begin{gathered}
\|f(z)\|_{X}^{p}=\sup _{\left\|x^{*}\right\| \leq 1}\left|\left(x^{*} \circ f\right)(z)\right|^{p} \leq \frac{1}{1-|z|^{2}} \sup _{\left\|x^{*}\right\| \leq 1}\left\|x^{*} \circ f\right\|_{H^{p}}^{p} \\
=\frac{1}{1-|z|^{2}}\|f\|_{w H^{p}(X)}^{p} .
\end{gathered}
$$


Consequently

$$
\begin{aligned}
\left\|u C_{\varphi} f\right\|_{H^{p}(X)}^{p} & =\sup _{0<r<1} \int_{\mathbb{T}}|u(r \zeta)|^{p}\|f(\varphi(r \zeta))\|_{X}^{p} d m(\zeta) \\
& \leq\|f\|_{w H^{p}(X)}^{p} \sup _{0<r<1} \int_{\mathbb{T}} \frac{|u(r \zeta)|^{p}}{1-|\varphi(r \zeta)|^{2}} d m(\zeta) .
\end{aligned}
$$

For our main results we need the following Dvoretzky's well-known theorem:

Lemma 2.2. [7] Suppose that $X$ is an infinite-dimensional complex Banach space. Then for any $\epsilon>0$ and $n \in \mathbb{N}$, there is a linear embedding $T_{n}: \ell_{2}^{n} \rightarrow X$ such that

$$
(1+\epsilon)^{-1}\left(\sum_{j=1}^{n}\left|a_{j}\right|^{2}\right)^{1 / 2} \leq\left\|\sum_{j=1}^{n} a_{j} T_{n} e_{j}\right\|_{X} \leq\left(\sum_{j=1}^{n}\left|a_{j}\right|^{2}\right)^{1 / 2}
$$

for any scalars $a_{1}, a_{2}, \cdots, a_{n}$ and some orthonormal basis $\left\{e_{1}, \cdots, e_{n}\right\}$ of $\ell_{2}^{n}$.

We also need the following lemma which is cited from [19, Theorem 2] and [8, Theorem 1].

Lemma 2.3. Suppose that $2 \leq p<\infty$, then

(i) The sequence $\left(k^{2 / p-1}\right)$ is a bounded coefficient multiplier $A^{2} \rightarrow A^{p}$.

(ii) The sequence $\left(k^{1 / p-1 / 2}\right)$ is a bounded coefficient multiplier $H^{2} \rightarrow H^{p}$.

Theorem 2.4 below provides a function-theoretic characterization of bounded weighted composition operators between Bergman spaces and this characterization is related to a Hilbert-Schmidt condition between the corresponding scalar spaces, see Liu and Yu [15].

Theorem 2.4. Let $X$ be any complex infinite-dimensional Banach space and $2 \leq p<\infty$. Then

$$
\left\|u C_{\varphi}: w A^{p}(X) \longrightarrow A^{p}(X)\right\| \approx\left(\int_{\mathbb{D}} \frac{|u(z)|^{p}}{\left(1-|\varphi(z)|^{2}\right)^{2}} d A(z)\right)^{1 / p} .
$$

In the case $p=2$, the equality holds.

Proof. From Proposition 2.1, it is sufficient to prove there exists a positive constant $c$ such that

$$
\left\|u C_{\varphi}: w A^{p}(X) \longrightarrow A^{p}(X)\right\| \geq c\left(\int_{\mathbb{D}} \frac{|u(z)|^{p}}{\left(1-|\varphi(z)|^{2}\right)^{2}} d A(z)\right)^{1 / p} .
$$

Suppose that $x \in X$ with $\|x\|=1$ and define $g: \mathbb{D} \rightarrow X$ by $g(z)=x$. Then $g$ is an analytic function on $\mathbb{D}$, and $\|g\|_{w A^{p}(X)}=1$, so that

$$
\left\|u C_{\varphi}\right\|^{p} \geq\|u g \circ \varphi\|_{A^{p}(X)}^{p}=\int_{\mathbb{D}}|u(z)|^{p} d A(z) .
$$

Hence

$$
\int_{\left\{z \in \mathbb{D}:|\varphi(z)|^{2}<1 / 2\right\}} \frac{|u(z)|^{p}}{\left(1-|\varphi(z)|^{2}\right)^{2}} d A(z) \leq 4 \int_{\mathbb{D}}|u(z)|^{p} d A(z) \leq 4\left\|u C_{\varphi}\right\|^{p} .
$$


So, it is sufficient to prove that there exists a positive constant $c$ such that

$$
\left\|u C_{\varphi}\right\|^{p} \geq c \int_{\left\{z \in \mathbb{D}:|\varphi(z)|^{2} \geq 1 / 2\right\}} \frac{|u(z)|^{p}}{\left(1-|\varphi(z)|^{2}\right)^{2}} d A(z) .
$$

Let $\lambda_{k}=k^{2 / p-1 / 2}$, for any $n \in \mathbb{N}$, we define functions $f_{n}$ as follows

$$
f_{n}(z)=\sum_{k=1}^{n} \lambda_{k} z^{k} T_{n} e_{k}
$$

where $T_{n}$ is the linear embedding in Lemma 2.2, and thus $\left\|T_{n}\right\|=1$ and $\left\|T_{n}^{-1}\right\| \leq$ $(1+\epsilon)$ and $\left(e_{1}, \cdots, e_{n}\right)$ is an orthonormal basis of $\ell_{2}^{n}$. Since for $2<p<\infty$ the sequence $\left(k^{2 / p-1}\right)$ is a coefficient multiplier $A^{2} \rightarrow A^{p}$, there exists $c>0$ such that for $x^{*} \in X^{*}$ with $\left\|x^{*}\right\| \leq 1$, we have

$$
\begin{aligned}
\left\|x^{*} \circ f_{n}\right\|_{A^{p}} & =\left\|\sum_{k=1}^{n} \lambda_{k} z^{k} x^{*} T_{n} e_{k}\right\|_{A^{p}} \\
& =\left\|\sum_{k=1}^{n} \lambda_{k} x^{*}\left(T_{n} e_{k}\right) z^{k}\right\|_{A^{p}} \\
& \leq c\left(\sum_{k=1}^{n}\left|x^{*}\left(T_{n} e_{k}\right)\right|^{2}\right)^{1 / 2} \leq c .
\end{aligned}
$$

It follows that $\left\|f_{n}\right\|_{w A^{p}(X)} \leq c$. Thus, Fatou's lemma implies that

$$
\begin{aligned}
\left\|u C_{\varphi}\right\|^{p} & \geq c^{-p} \limsup _{n \rightarrow \infty}\left\|u C_{\varphi} f_{n}\right\|_{A^{p}(X)}^{p} \\
& =c^{-p} \limsup _{n \rightarrow \infty} \int_{\mathbb{D}}|u(z)|^{p}\left\|\sum_{k=1}^{n} \lambda_{k} \varphi(z)^{k} T_{n} e_{k}\right\|_{X}^{p} d A(z) \\
& \geq \frac{c^{-p}}{(1+\epsilon)^{p}} \limsup _{n \rightarrow \infty} \int_{\mathbb{D}}\left(\sum_{k=1}^{n} k^{4 / p-1}|\varphi(z)|^{2 k}\right)^{p / 2}|u(z)|^{p} d A(z) \\
& =\frac{c^{-p}}{(1+\epsilon)^{p}} \int_{\mathbb{D}}\left(\sum_{k=1}^{\infty} k^{4 / p-1}|\varphi(z)|^{2 k}\right)^{p / 2}|u(z)|^{p} d A(z) \\
& \geq \frac{c_{1} c^{-p}}{(1+\epsilon)^{p}} \int_{\left\{z \in \mathbb{D}:|\varphi(z)|^{2} \geq 1 / 2\right\}} \frac{|u(z)|^{p}}{\left(1-|\varphi(z)|^{2}\right)^{2}} d A(z)
\end{aligned}
$$

and the last inequality is derived by Lemma 2.3 [13]. As $\epsilon>0$ is arbitrary, we obtain the desired lower bound estimate.

In case $p=2$, change the functions $f_{n}$ as follows

$$
f_{n}(z)=\sum_{k=0}^{n-1} \sqrt{k+1} z^{k} T_{n} e_{k}
$$


Since $\left((k+1)^{1 / 2} z^{k}\right)$ is orthonormal in $A^{2},\left\|x^{*} \circ f_{n}\right\|_{A^{2}}^{2} \leq 1$, for any $x^{*} \in X^{*}$ with $\left\|x^{*}\right\| \leq 1$. By the same argument as in the case $2<p<\infty$, we can get

$$
\begin{aligned}
\left\|u C_{\varphi}\right\|^{2} & \geq \frac{1}{(1+\epsilon)^{2}} \int_{\mathbb{D}} \sum_{k=0}^{\infty}(k+1)|\varphi(z)|^{2 k}|u(z)|^{2} d A(z) \\
& \geq \frac{1}{(1+\epsilon)^{2}} \int_{\mathbb{D}} \frac{|u(z)|^{2}}{\left(1-|\varphi(z)|^{2}\right)^{2}} d A(z) .
\end{aligned}
$$

As $\epsilon \rightarrow 0$, the claim follows immediately.

The following theorem provides a function-theoretic characterization of bounded weighted composition operators between Hardy spaces and this characterization is related to a Hilbert-Schmidt condition between the corresponding scalar spaces, refer to Matache [16].

Theorem 2.5. Let $X$ be any complex infinite-dimensional Banach space and $2 \leq p<\infty$. Then

$$
\left\|u C_{\varphi}: w H^{p}(X) \longrightarrow H^{p}(X)\right\| \approx\left(\int_{\mathbb{T}} \frac{|u(\zeta)|^{p}}{1-|\varphi(\zeta)|^{2}} d m(\zeta)\right)^{1 / p} .
$$

In the case $p=2$, the equality holds.

Proof. If $u=0$, then the result is obvious. So we may set $u \neq 0$. If

$$
\int_{\mathbb{T}} \frac{|u(\zeta)|^{p}}{1-|\varphi(\zeta)|^{2}} d m(\zeta)<\infty
$$

then $|\varphi(\zeta)|<1$ a.e. $\zeta \in \mathbb{T}$, so that $\left(1-|\varphi(\zeta)|^{2}\right)^{-1}=\sum_{n=0}^{\infty}|\varphi(\zeta)|^{2 n}$ a.e. on $\mathbb{T}$.

Monotone convergence and the subharmonicity of $\left|u^{p}(\cdot) \varphi^{2 n}(\cdot)\right|$ imply that

$$
\begin{aligned}
\int_{\mathbb{T}} \frac{|u(\zeta)|^{p}}{1-|\varphi(\zeta)|^{2}} d m(\zeta) & =\sum_{n=0}^{\infty} \sup _{0<r<1} \int_{\mathbb{T}}|u(r \zeta)|^{p}|\varphi(r \zeta)|^{2 n} d m(\zeta) \\
& \geq \sup _{0<r<1} \int_{\mathbb{T}} \frac{|u(r \zeta)|^{p}}{1-|\varphi(r \zeta)|^{2}} d m(\zeta) .
\end{aligned}
$$

So, the upper bound estimate is obtained from Proposition 2.1.

For the lower bound estimate, if $\left\|u C_{\varphi}: w H^{p}(X) \rightarrow H^{p}(X)\right\|$ is finite, we first note that

$$
\left\|u C_{\varphi}\right\|^{p} \geq\|u g \circ \varphi\|_{H^{p}(X)}^{p}=\|u\|_{H^{p}}^{p}
$$

where $g(z)=x \in X$ with $\|x\|=1$. So $u$ has a.e. radial limits $u(\zeta)=\lim _{r \rightarrow 1^{-}} u(r \zeta)$ on $\mathbb{T}$. Again we have

$$
\int_{\left\{\zeta \in \mathbb{T}:|\varphi(r \zeta)|^{2}<1 / 2\right\}} \frac{|u(r \zeta)|^{p}}{1-|\varphi(r \zeta)|^{2}} d m(\zeta)<2\|u\|_{H^{p}}^{p} \leq 2\left\|u C_{\varphi}\right\|^{p} .
$$

So we only need to prove that there exists $c>0$ such that

$$
\left\|u C_{\varphi}\right\|^{p} \geq c \int_{\left\{\zeta \in \mathbb{T}:|\varphi(r \zeta)|^{2} \geq 1 / 2\right\}} \frac{|u(r \zeta)|^{p}}{1-|\varphi(r \zeta)|^{2}} d m(\zeta) .
$$


Let $\lambda_{k}=k^{1 / p-1 / 2}$ and define

$$
f_{n}(z):=\sum_{k=1}^{n} \lambda_{k} z^{k} T_{n} e_{k}
$$

where the linear embedding $T_{n}$ is the same as in Lemma 2.2, $\left\|T_{n}\right\|=1$ and $\left\|T_{n}^{-1}\right\| \leq(1+\epsilon)$ and $\left(e_{1}, \cdots, e_{n}\right)$ is an orthonormal basis of $\ell_{2}^{n}$. Since for $2<p<$ $\infty$ the sequence $\left(\lambda_{k}\right)$ is a bounded coefficient multiplier $H^{2} \rightarrow H^{p}$, there exists $c>0$ such that for $x^{*} \in X^{*}$ with $\left\|x^{*}\right\| \leq 1$, we have

$$
\left\|x^{*} \circ f_{n}\right\|_{H^{p}}=\left\|\sum_{k=1}^{n} \lambda_{k} x^{*}\left(T_{n} e_{k}\right) z^{k}\right\|_{H^{p}} \leq c\left(\sum_{k=1}^{n}\left|x^{*}\left(T_{n} e_{k}\right)\right|^{2}\right)^{1 / 2} \leq c .
$$

Thus $\left\|f_{n}\right\|_{w H^{p}(X)} \leq c$ and by using Fatou's lemma and Lemma 2.3 [13], we have

$$
\begin{aligned}
\left\|u C_{\varphi}\right\|^{p} & \geq c^{-p} \limsup _{n \rightarrow \infty}\left\|u C_{\varphi} f_{n}\right\|_{H^{p}(X)}^{p} \\
& \geq c^{-p} \limsup _{n \rightarrow \infty} \int_{\mathbb{T}}|u(r \zeta)|^{p}\left\|\sum_{k=1}^{n} \lambda_{k} \varphi(r \zeta)^{k} T_{n} e_{k}\right\|_{X}^{p} d m(\zeta) \\
& \geq \frac{c^{-p}}{(1+\epsilon)^{p}} \limsup _{n \rightarrow \infty} \int_{\mathbb{T}}\left(\sum_{k=1}^{n} k^{2 / p-1}|\varphi(r \zeta)|^{2 k}\right)^{p / 2}|u(r \zeta)|^{p} d m(\zeta) \\
& =\frac{c^{-p}}{(1+\epsilon)^{p}} \int_{\mathbb{T}}\left(\sum_{k=1}^{\infty} k^{2 / p-1}|\varphi(r \zeta)|^{2 k}\right)^{p / 2}|u(r \zeta)|^{p} d m(\zeta) \\
& \geq \frac{c_{1} c^{-p}}{(1+\epsilon)^{p}} \int_{\left\{\zeta \in \mathbb{T}:|\varphi(r \zeta)|^{2} \geq 1 / 2\right\}} \frac{|u(r \zeta)|^{p}}{1-|\varphi(r \zeta)|^{2}} d m(\zeta) .
\end{aligned}
$$

Take $\epsilon=1$, then

$$
\left\|u C_{\varphi}\right\|^{p} \geq c \int_{\mathbb{T}} \frac{|u(r \zeta)|^{p}}{1-|\varphi(r \zeta)|^{2}} d m(\zeta)
$$

As $r \rightarrow 1$,

$$
\begin{aligned}
\left\|u C_{\varphi}\right\|^{p} & \geq c \limsup _{r \rightarrow 1} \int_{\mathbb{T}} \frac{|u(r \zeta)|^{p}}{1-|\varphi(r \zeta)|^{2}} d m(\zeta) \\
& \geq c \int_{\mathbb{T}} \frac{|u(\zeta)|^{p}}{1-|\varphi(\zeta)|^{2}} d m(\zeta) .
\end{aligned}
$$

In case $p=2$, change the functions $f_{n}$ as

$$
f_{n}(z):=\sum_{k=1}^{n} z^{k-1} T_{n} e_{k}
$$

Since $\left(z^{k}\right)$ is orthonormal in $H^{2},\left\|x^{*} \circ f_{n}\right\|_{H^{2}}^{2} \leq 1$, for any $x^{*} \in X^{*}$ with $\left\|x^{*}\right\| \leq 1$. The rest of the proof is similar to those of previous theorem.

By taking $u(z) \equiv 1$, we have the following corollary which is the Theorems 2.2 and 3.2 of [13], for $2 \leq p<\infty$. 
Corollary 2.6. Let $X$ be any complex infinite-dimensional Banach space and $2 \leq p<\infty$. Then

$$
\left\|C_{\varphi}: w A^{p}(X) \longrightarrow A^{p}(X)\right\| \approx\left(\int_{\mathbb{D}} \frac{1}{\left(1-|\varphi(z)|^{2}\right)^{2}} d A(z)\right)^{1 / p}
$$

and

$$
\left\|C_{\varphi}: w H^{p}(X) \longrightarrow H^{p}(X)\right\| \approx\left(\int_{\mathbb{T}} \frac{1}{1-|\varphi(\zeta)|^{2}} d m(\zeta)\right)^{1 / p} .
$$

Letting $\varphi(z) \equiv z$ (it is impossible in (2.1), since the finiteness in the righthand side of $(2.1)$ implies $|\varphi(\zeta)|<1$ a.e. $\zeta \in \mathbb{T}$ except $u(z)=0)$, we obtain the corresponding result for the multiplication operator.

Corollary 2.7. Let $X$ be any complex infinite-dimensional Banach space and $2 \leq p<\infty$. Then

$$
\left\|M_{u}: w A^{p}(X) \longrightarrow A^{p}(X)\right\| \approx\left(\int_{\mathbb{D}} \frac{|u(z)|^{p}}{\left(1-|z|^{2}\right)^{2}} d A(z)\right)^{1 / p} .
$$

Acknowledgement. The authors wish to express their gratitude to the anonymous referee for giving valuable comments and suggestions, which have improved this paper. The third author was supported by NSFC(11271293).

\section{REFERENCES}

1. J. Arregui and O. Blasco, Bergman and Bloch spaces of vector-valued functions, Math Nachr. 261/262 (2003), 3-22.

2. O. Blasco, Boundary values of vector-valued harmonic functions considered as operators, Studia Math. 86 (1987), 19-33.

3. O. Blasco, Introduction to vector valued Bergman spaces, in Function Spaces and Operator Theory (Joensuu, 2003), Univ. Joensuu Dept. Math. Rep. Ser., 8, Univ. Joensuu, Joensuu (2005), 9-30.

4. J. Bonet, P. Domanski and M. Lindstrom, Weakly compact composition operators on analytic vector-valued function spaces, Ann. Acad. Sci. Fenn. Math. 26 (2001), 233-248.

5. M.D. Contreras and A.G. Hernandez-Diaz, Weighted composition operators on Hardy spaces, J. Math. Anal. Appl. 263 (2001), no. 1, 224-233.

6. C.C. Cowen and B.D. Maccluer, Composition operators on spaces of analytic functions, Studies in Advanced Mathematics, CRC Press, Boca Raton, Fla, USA, 1995.

7. J. Diestel, H. Jarchow and A. Tonge, Absolutely Summing Operators, Cambridge Univ. Press, Cambridge, 1995.

8. P.L. Duren, On the multipliers of $H^{p}$ spaces, Proc. Amer. Math. Soc. 22 (1969), 24-27.

9. P.L. Duren, Theory of $H^{p}$ spaces, Academic Press, New York, 1970.

10. K. Esmaeili and H. Mahyar, Weighted composition operators between vector-valued Lipschitz function spaces, Banach J. Math. Anal. 7 (2013), no. 1, 59-72.

11. J. Laitila, Weakly compact composition operators on vector-valued BMOA, J. Math. Anal. Appl. 308 (2005), 730-745.

12. J. Laitila and H.-O. Tylli, Operator-weighted composition operators on vector-valued analytic function spaces, Illinois J. Math. 53 (2009), 1019-1032.

13. J. Laitila, H.-O. Tylli and M. Wang, Composition operators from weak to strong spaces of vector-valued analytic functions, J. Operator Theory 62 (2009), 281-295.

14. P. Liu, E. Saksman and H.-O. Tylli, Small composition operators on analytic vector-valued function spaces, Pacific J. Math. 184 (1998), 295-309. 
15. Y. Liu and Y. Yu, Schatten class weighted composition operators on the Bergman space of the unit ball, J. Math. Res. Exposition 27 (2007), no. 3, 533-538.

16. V. Matache, Weighted Composition Operators on $\mathrm{H}^{2}$ and Applications, Complex Anal. Oper. Theory 2 (2008), 169-197.

17. J.H. Shapiro, Composition operator and classical function theory, Springer-Verlag, New York, 1993.

18. A.K. Sharma and S. Ueki, Composition operators between weighted Bergman spaces with admissible Békollé weights, Banach J. Math. Anal. 8 (2014), no. 1, 64-88.

19. D. Vukotić, On the coefficient multipliers of Bergman spaces, J. London Math. Soc. 50 (1994), 341-348.

20. D. Vukotić, A sharp estimate for $A_{\alpha}^{p}$ function in $\mathbb{C}^{n}$, Proc. Amer. Math. Soc. 117 (1993), $753-756$.

21. M. Wang, Weighted composition operators between Dirichlet spaces, Acta Math. Sci. 31B (2011), no. 2, 641-651.

22. E. Wolf, Bounded, compact and Schatten class weighted composition operators between weighted Bergman spaces, Commun. Korean Math. Soc. 26 (2011), no. 3, 455-462.

23. K. Zhu, Spaces of holomorphic functions in the unit ball, Springer, New York, 2005.

${ }^{1}$ FACULTy OF MATHEMATICAL SCIENCES, UNIVERSity OF TABRIZ, P.O.BOX: 5166615648, TABRIZ, IRAN.

E-mail address: m_hasanloo@tabrizu.ac.ir

E-mail address: hvaezi@tabrizu.ac.ir

${ }^{2}$ School of Mathematics and Statistics, Wuhan University, Wuhan 430072, China.

E-mail address: mfwang.math@whu.edu.cn 